\title{
On Rational Elliptic Surfaces with Multiple Fibers
}

\author{
By \\ Yoshio Fujimoto*
}

\section{§0. Introduction}

It is well-known that a rational elliptic surface with global sections is a nine-pointsblowing-up of $\mathbf{p}^{2}$ which may be infinitely near and can be obtained from the pencil of cubic curves in $\mathbf{P}^{2}$. This object has been studied by Demauzuer-Pinkham [1], Looijenga [4] and Naruki [5] [6].

In this paper, we shall study the structure of rational elliptic surfaces with multiple fibers. A rational elliptic surface with multiple fibers of multiplicity $m$ can be obtained from a rational elliptic surface with global sections by performing logarithmic transformations at one point on the base curve $\mathbb{P}^{1}$. It can also be blown down to $\mathbb{P}^{2}$. In this case, if the nine points $p_{1}, p_{2}, \ldots, p_{9}$ on $\mathbb{P}^{2}$ are mutually distinct and the multiple fiber is of type ${ }_{m} I_{0}$, it is obtained from the pencil generated by $m$-fold cubic which passes through $P_{i}^{\prime}$ 's and a curve of degree $3 m$ which has an ordinary singularity of multiplicity $m$ at each $P_{i}(1 \leq i \leq 9)$ and is non-singular outside them.

The method of this paper is to study birational geometry of curves in $\mathbb{P}^{2}$ through the logarithmic transformations.

Our main result is as follows.

Main Theorem (2.1.) Let $C$ be a non-singular cubic curve in $\mathbf{P}^{2}$ with the fixed inflexion point $Q$ on $C$ such that $C$ should be given the natural group structure with $Q$ as the identity. Fix such an isomorphism $\varepsilon: C \simeq T$, where $T$ is a one-dimensional complex torus. Take nine points $p_{i}(1 \leq i \leq 9)$ on $C$ (which may be infinitely near) and let $S$ be a surface obtained by blowing up $\mathbb{P}^{2}$ at $p_{i}$ 's $(1 \leq i \leq 9$.) Then

(1) S has the structure of an elliptic surface with global sections if and only if $\sum_{i=1}^{9} \varepsilon\left(p_{i}\right)=0$, where \pm means the additive group law in a complex torus $T$.

(2) $S$ has the structure of an elliptic surface with one multiple fiber of multiplicity $m$ if and only if $\sum_{i=1}^{9} \varepsilon\left(p_{i}\right)$ is of order $m$ in $T$. And there is a one-to-one correspondence between $\sum_{i=1}^{9} \varepsilon\left(p_{i}\right)$ and the normal bundle of the support of the multiple fiber in $S$.

Communicated by K. Saito, May 19, 1988.

* Department of Mathematics, Shizuoka University, Ohya, Shizuoka, Japan.

Research supported by the JSPS Fellowships for Japanese Junior Scientists. 
From this theorem, we easily prove that if we blow up $\mathbb{P}^{2}$ at nine points in a sufficiently general position, it does not have the structure of an elliptic surface in any way.

The construction of the paper is as follows. In $\S 1$, we shall consider the structure of rational elliptic surface with multiple fibers. (cf. theorem (1.1.)) In $\S 2$, we shall prove our main theorem (2.1.). In $\S 3$, we shall construct a family of rational elliptic surfaces with multiple fibers after Naruki's method. (cf. [6])

The author wishes to express his sincere thanks to Professors $\mathbb{K}$. Ueno and $\mathbb{I}$. Naruki for many useful suggestions.

\section{Notation and Convention}

By an elliptic fiber space $f: V \rightarrow W$, we mean that $f$ is a proper surjective morphism of a complex manifold $V$ to a complex manifold $W$, where each fiber is connected and the general fibers are non-singular elliptic curves. In particular, when $W$ is a surface and $V$ is a three dimensional complex manifold, we say that $V$ is an elliptic threefold over $W$. Here, "surface" means a two dimensional (not necessarily compact) complex manifold.

For a compact complex manifold $X$, we use the following notation.

$N_{V / X}$ : the normal bundle of $V$ in $X$, where $V$ is a submanifold of $X$. $\kappa(X)$ : the Kodaira dimension of $X$.

$K_{X}$ : The canonical bundle of $X$.

$h^{p, q}=\operatorname{dim}_{\mathrm{C}} H^{q}\left(X, \Omega_{X}^{p}\right)$

$q(X)=\operatorname{dim}_{\mathbf{C}} H^{1}\left(X, \mathcal{O}_{X}\right)$

$(X)^{n}:=\underbrace{X \times \cdots \times X}$

$n$ times direct product

$\Sigma_{n}:=\mathbb{P}\left(\mathcal{O}_{\mathbf{P}^{1}} \oplus \mathcal{O}_{\mathbb{P}^{1}}(n)\right)$

$X$ is in the class $\mathscr{C}$ in the sense of Fujiki if $X$ is a meromorphic image of a compact Kähler manifold.

If $D$ is a divisor on $X$, we set

$[D]$ : the line bundle on $X$ determined by $D$.

\section{§1. Preliminaries}

Proposition (1.1.) Let $f: S \rightarrow \mathbb{P}^{1}$ be a relatively minimal rational elliptic surface. Then $S$ can be obtained as a nine-points blowing-up of $\mathbb{P}^{2}$ whose center may be infinitely near and the structure of the elliptic fibration is one of the followings:

(1) If $f: S \rightarrow \mathbb{P}^{1}$ has a global section, $S$ can be obtained from the pencil generated by cubic curves in $\mathbb{P}^{2}$ and the anti-canonical map $\Phi_{\left|-K_{S}\right|}: S \rightarrow \mathbb{P}^{1}$ is isomorphic to the original elliptic fibration.

(2) If $f: S \rightarrow \mathbb{P}^{1}$ has only one multiple fiber of multiplicity $m(\geq 2), S$ can be obtained from a rational elliptic surface $X$ with global sections by performing 
logarithmic transformations, where $m$ and $X$ are uniquely determined by $S$. And the anti-pluricanonical map $\Phi_{\left|-m K_{s}\right|}: S \rightarrow \mathbb{P}^{1}$ is isomorphic to the original elliptic fibration. Moreover, if the nine points $p_{i}(1 \leq i \leq 9)$, which are the center of blowings-up, are all distinct, and the multiple fiber is of type ${ }_{m} I_{0}, S$ can be obtained from the pencil generated by $m$-fold cubic $m E$ and $C$, where $E$ is a non-singular cubic curve in $\mathbb{P}^{2}$ and $C$ is a singular curve of degree $3 m$ in $\mathbb{P}^{2}$, which has an ordinary singularity of multiplicity $m$ at each $p_{i} \in E(1 \leq i \leq 9)$ and is non-singular outside them.

Proof. If $f: S \rightarrow \mathbb{P}^{1}$ has no multiple fibers, $S$ has a global section (since $S$ is rational) and the result is well-known. (See Naruki [5].) Hence we treat the case where $f: S \rightarrow \mathbb{P}^{1}$ has multiple fibers.

Step 1. First, we show that $f: S \rightarrow \mathbb{P}^{1}$ has only one multiple fiber $m E$. Assume that $S$ has $l$ multiple fibers $m_{i} E_{i}(1 \leq i \leq l)$ of multiplicity $m_{i}$. Since $S$ is rational, $\chi\left(\mathcal{O}_{S}\right)=1$, the canonical bundle formula of Kodaira [3] implies

where

$$
K_{S} \simeq f^{*} \mathcal{O}_{\mathbb{P} 1}(-1)+\sum_{i=1}^{l}\left(m_{i}-1\right)\left[E_{i}\right],
$$

Since

we have

$$
f^{*} \mathcal{O}_{\mathbf{P} 1}(1) \simeq\left[m_{i} E_{i}\right]
$$

$$
\kappa(S)=-\infty,
$$

$$
-1+\sum_{i=1}^{l}\left(1-1 / m_{i}\right) \leq 0 .
$$

From this inequality, we have either

$$
l=2 \quad \text { and } \quad\left(m_{1}, m_{2}\right)=(2,2)
$$

or

$$
l=1 .
$$

However in the case (a), we see that $2 K_{S} \simeq \mathcal{O}_{S}$ and $S$ is an Enriques surface. Hence the claim follows.

Since

$$
K_{S} \sim f^{*} \mathcal{O}_{\mathbf{P} 1}(-1)+(m-1)[E],
$$

and

$$
f^{*} \mathcal{O}_{\mathbf{P}^{1}}(1) \simeq m[E],
$$

we have

$$
K_{S} \sim-[E]
$$

and

$$
\Phi_{\left|-m K_{s}\right|}: S \rightarrow \mathbb{P}^{1}
$$

is isomorphic to the original elliptic fibration up to projective equivalence of the base curve $\mathbb{P}^{1}$. 
Step 2. We show that $S$ can be obtained as a nine-points blowing up of $\mathbb{P}^{2}$. For since $-m K_{S}=f^{*} \mathcal{O}_{\mathbb{P}^{1}}(1)$, we have the following inequality for any non-singular rational curve $C$ on $S$ :

$$
C^{2}=C \cdot(-K)-2 \geq-2
$$

The same inequality holds for any blowing down of $S$, hence $S$ can be blown down to $\mathbb{P}^{2}$ or $\mathbb{P}^{1} \times \mathbb{P}^{1}$ or $\Sigma_{2}$. Clearly every one-point-blowing-up of $\mathbb{P}^{1} \times \mathbb{P}^{1}$ is a two-pointsblowing-up of $\mathbb{P}^{2}$. Moreover we see from the above inequality that for the last case, the center of the blowing-up is disjoint from the (-2)-curve of $\Sigma_{2}$, and such a blowingup can be blown down to $\mathbb{P}^{2}$ by means of elementary transformations. Hence the claim follows.

Step 3. By Kodaira [3], $S$ can be obtained from an elliptic surface $X$ free from multiple fibers by means of logarithmic transformations. Since the irregularity is invariant under logarithmic transformations, we have $q(X)=q(S)=0$ and $h^{1,0}(X) \leq$ $q(X)=0$. Hence $q(X)=h^{1,0}(X)=0$ and $X$ is Kähler. Since $\kappa(X) \leq \kappa(S)$, we have $\kappa(X)=-\infty$ and from the classification theory of surfaces [3], $X$ is a rational elliptic surface with global sections.

Step 4. Let $l$ be the total transform of a line in $\mathrm{P}^{2}$ and $e_{i}(1 \leq i \leq 9)$ be exceptional curves. Then $\operatorname{Pic}(S) \simeq \mathbb{Z}^{10}$, generated by $l, e_{1}, \ldots, e_{9}$ and the intersection pairing on $S$ is given by

$$
l^{2}=1, \quad e_{i}^{2}=-1, \quad l e_{i}=0, \quad e_{i} e_{j}=0 \quad \text { for } \quad i \neq j .
$$

The canonical bundle of $S$ is given by:

$$
K_{S} \sim-3 l+e_{1}+\cdots+e_{9} .
$$

Hence from $(*)$ and $(* *)$, we have:

$$
E \sim 3 l-\sum_{i=1}^{9} e_{i} \sim-K_{S}
$$

Assume that the nine points $p_{i}(1 \leq i \leq 9)$, which are the center of the blowing-up, are all distinct and the multiple fiber $m E$ is of type ${ }_{m} I_{0}$. Then from remark (1.2.) below, the image of $E$ by this blowing-down is a non-singular cubic curve in $\mathbb{P}^{2}$. Since $\operatorname{dim}|i E|=0$ for $1 \leq i \leq m-1$ and $\operatorname{dim}|m E|=1$, by remark (1.2.), there exists a singular curve $C$ of degree $3 m$ in $\mathbb{P}^{2}$ which has an ordinary singularity of multiplicity $m$ at each $p_{i} \in E(1 \leq i \leq 9)$ and is non-singular outside them. Conversely let $L$ be the pencil generated by such $m E$ and $C$ and consider the rational map

$$
\Phi_{|L|}: \mathbb{P}^{2} \rightarrow \mathbb{P}^{1}
$$

associated to $L$. By easy calculation, we see that the normalization of $C$ is a nonsingular elliptic curve.

By blowing up at $P_{i}^{\prime}$ 's, which are the base points of the pencil $\mathbb{L}$, all members of the pencil can be separated and $\Phi_{|L|}$ extends to a holomorphic map $\varphi: S \rightarrow \mathbb{P}^{1}$ which is isomorphic to the anti-pluricanonical map $\Phi_{\left|-m K_{s}\right|}: S \rightarrow \mathbb{P}^{1}$ (hence to the original elliptic 
fibration up to projective equivalence of the base curve $\mathbb{P}^{1}$.) Note that $m$ is uniquely determined as the smallest positive integer such that $\operatorname{dim}\left|-m K_{S}\right|=1$ and so the case (1) and (2) cannot occur simultaneously.

q.e.d.

Remark (1.2.) In the case (1), the (-1)-curve $e$ is a global section of the elliptic fibration. However in the case (2), the (-1)-curve is an $m$-section, that is, $e$ is an $m$-sheeted coverings of $\mathbb{P}^{1}$ ramifying over the point where the multiple fiber lie and with the ramification index $m$.
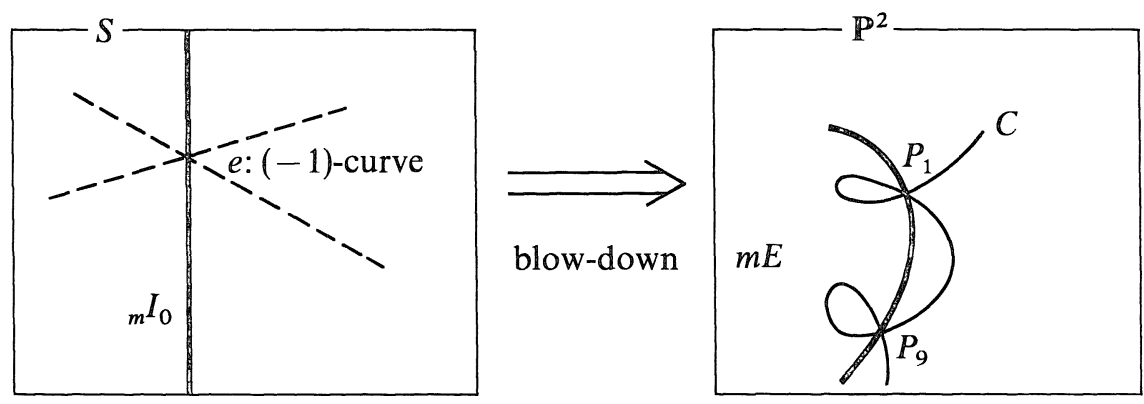

Remark (1.3.) When $m=2$, the pencil of nine-nodal sextics containing one double cubic was classically called a Halphen pencil. (cf. [7])

\section{§2. Proof of Main Theorem (2.1.)}

In this section, we shall prove our main Theorem (2.1.).

Remark (2.2.) Note that the condition in Theorem (2.1.) is independent of the choice of the inflexion point $Q$.

First, we prove (1) and the necessary condition in (2).

Proof of (1). Necessity. Assume that $\varphi: S \rightarrow \mathbb{P}^{1}$ is a rational elliptic surface with global sections. Then $S$ is a nine-points-blowing-up of $\mathbb{P}^{2}$ and we have: $-K_{S} \simeq \varphi^{*} \mathcal{O}_{\mathbb{P}^{1}}(1) \simeq 3 l-\sum_{i=1}^{9} e_{i}$, where we use the same notation as in the proof of proposition (1.1.). Since $\operatorname{dim}\left|3 l-\sum_{i=1}^{9} e_{i}\right|=1$, there exists a pencil of cubic curves passing through the nine points $p_{1}, p_{2}, \ldots, p_{9}$. For a generic member $C_{t}$ of the pencil, we have $\left.\left.C_{t}\right|_{C} \simeq \mathcal{O}_{\mathbb{P}^{2}}(3)\right|_{C}$, hence $\sum_{i=1}^{9} p_{i} \sim 9 Q$. Then from Abel's theorem, we have $\sum_{i=1}^{9} \varepsilon\left(p_{i}\right)=0$.

Sufficiency. Conversely assume that $\sum_{i=1}^{9} \varepsilon\left(p_{i}\right)=0$. Then from Abel's theorem, we have $\sum_{i=1}^{9} p_{i} \sim 9 Q$ in $\operatorname{Pic}(C)$. By the exact sequence

$$
\begin{gathered}
\left.0 \rightarrow \mathcal{O}_{\mathbf{P}^{2}} \rightarrow \mathcal{O}_{\mathbb{P}^{2}}(3) \rightarrow \mathcal{O}_{\mathbf{P}^{2}}(3)\right|_{C} \rightarrow 0, \\
\mid \mathrm{R} \\
\mathcal{O}_{C}(9 Q)
\end{gathered}
$$


we have:

$$
0 \rightarrow \mathbb{C} \rightarrow \Gamma\left(\mathbb{P}^{2}, \mathcal{O}_{\mathbb{P}^{2}}(3)\right) \rightarrow \Gamma\left(\mathbb{P}^{2}, \mathcal{O}_{C}(9 Q)\right) \rightarrow 0 .
$$

Therefore there exists a pencil of cubic curves in $\mathbb{P}^{2}$ passing through the nine points $p_{i}$ $(1 \leq i \leq 9)$ and the claim follows.

q.e.d.

Proof of (2). Necessity. We use the same notation as in the proof of Proposition (1.1.). Let $\varphi: S \rightarrow \mathbb{P}^{1}$ be a rational elliptic surface with one multiple fiber $m E$ of multiplicity $m$. Then $S$ can be obtained by blowing-up at nine points $p_{1}, \ldots, p_{9}$ on $\mathbb{C}$, which is a non-singular cubic curve in $\mathbb{P}^{2}$.

Since $E \in\left|-K_{S}\right|$ is the fixed component of $\left|-K_{S}\right|, E$ coincides with the strict transform of $C$ and we have:

$$
E \sim 3 l-\sum_{i=1}^{9} e_{i} \sim-K_{S}
$$

Since $S$ has a multiple fiber, it follows from (1) in Theorem (2.1.) that there exists an inflexion point $Q_{0}$ of $C$ which does not coincide with any $p_{i}(1 \leq i \leq 9)$. From Remark (2.2.), we may assume that $Q=Q_{0}$.

Choose $l$ as a tangent line of $C$ at $Q_{0}$. Then from (a), we have:

$$
\left.[E]\right|_{E} \sim 9 Q_{0}-\sum_{i=1}^{9} p_{i}
$$

On the other hand, by Kodaira [3], $\left.[E]\right|_{E}$ is of order $m$ in $\operatorname{Pic}^{0}(E)$. Hence from Abel's theorem, $\sum_{i=1}^{9} \varepsilon\left(p_{i}\right)$ is an $m$-torsion point of $T$.

q.e.d.

Corollary (2.3.) Let $B$ be the subset of $\left(\mathbb{P}^{2}\right)^{9}:=\mathbb{P}^{2} \times \cdots \times \mathbb{P}^{2}$ consisting of points

9 times

for which the following condition is satisfied.

Condition ( $A$ ). The rational surface $S$, obtained by blowing up $\mathbb{P}^{2}$ at nine points $p_{1}, p_{2}, \ldots, p_{9}$, does not have the structure of an elliptic surface.

Then $B$ is dense in $\left(\mathbb{P}^{2}\right)^{9}$ in the complex topology.

Proof. For any $\left(Q_{1}, \ldots, Q_{9}\right) \in\left(\mathbb{P}^{2}\right)^{9}$, let $U$ be an arbitrary small open neighborhood of $\left(Q_{1}, \ldots, Q_{9}\right)$. Then there exists $\left(p_{1}, \ldots, p_{9}\right) \in U$ such that the cubic curve in $\mathbb{P}^{2}$ passing through nine points $p_{i}(1 \leq i \leq 9)$ is unique and non-singular. (We call it $\mathbb{C}$ and give the natural group structure.) Next, take nine points $\left(R_{1}, \ldots, R_{9}\right) \in(C)^{9} \cap U$ such that $\sum_{i=1}^{9} R_{i}$ is of infinite order in $C$.

Then from the necessity part of (2) in Theorem (2.1.), $\left(R_{1}, \ldots, R_{9}\right)$ satisfies the condition (A) and we have $U \cap B \neq \phi$.

Remark (2.4.) $B$ is not open in the complex topology, as we will see later.

Proof of sufficiency of Theorem (2.1.) Assume that $\eta:=\sum_{i=1}^{9} \varepsilon\left(p_{i}\right)$ is of order $m$ 
in $T$. Then we can take the corresponding ètale covering $\widetilde{T}$ of $T$. Fix an isomorphism $\tilde{\varepsilon}: \widetilde{C} \simeq \widetilde{T}$ with $\varepsilon(\widetilde{Q})=0$, where $\widetilde{C}$ is a nonsingular cubic curve in $\mathbb{P}^{2}$ with the fixed inflexion point $\widetilde{Q}$ on $\tilde{C}$. The family $\mathbb{P}^{2} \times \widetilde{C}^{8} \rightarrow \widetilde{C}^{8}:=\widetilde{C} \times \cdots \times \widetilde{C}$ has nine 8 times

sections $\sigma_{j}(1 \leq j \leq 9)$, defined by:

$$
\begin{array}{cc}
\sigma_{j}\left(Q_{1}, \ldots, Q_{8}\right) & =\left(l\left(Q_{j}\right), Q_{1}, \ldots, Q_{8}\right) \quad(1 \leq j \leq 8) \\
\sigma_{9}\left(Q_{1}, \ldots, Q_{8}\right) & =\left(l\left(-\left(Q_{1}+\cdots+Q_{8}\right)\right), Q_{1}, \ldots, Q_{8}\right),
\end{array}
$$

where $l: \widetilde{C} \rightarrow \mathbb{P}^{2}$ is an inclusion and \pm means the additive group law in a complex torus $\tilde{T}$.

Blow up $\mathbb{P}^{2} \times \widetilde{C}^{8}$ with the center of the image of $\sigma_{1}$. Then we have:

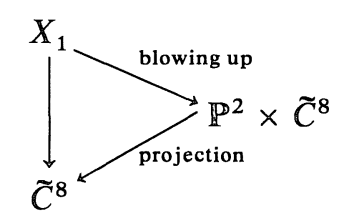

Next, blow up $X_{1}$ with the center of the strict transform of $\sigma_{2}$ in $X_{1}$ and we obtain $X_{2}$. Continuing this process, we obtain $X:=X_{9}$, which is a family of surfaces obtained by blowing up $\mathbb{P}^{2} \times \widetilde{C}^{8}$ with the center of nine sections $\sigma_{1}, \ldots, \sigma_{9}$.

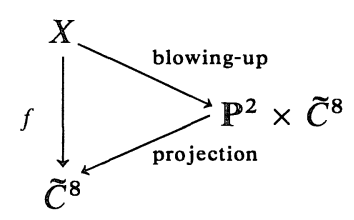

Clearly $f: X \rightarrow \widetilde{C}^{8}$ is proper and smooth and each fiber of $f$ is a nine-pointsblowing-up of $\mathbb{P}^{2}$ which may be infinitely near. Then from our choice of nine sections $\sigma_{j}(1 \leq j \leq 9)$, we have the following commutative diagram by Proposition (1.1.) and Theorem (2.1.) (1):

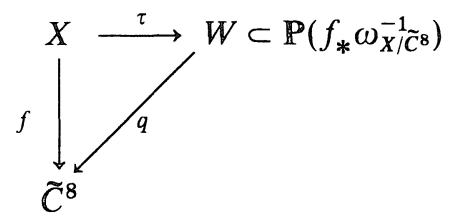

where

(1) $\tau: X \rightarrow W \subset \mathbb{P}\left(f_{*} \omega_{X / \widetilde{C}^{8}}^{-1}\right)$ is a morphism and $X$ is an elliptic fiber space over $W$.

(2) $q: W \rightarrow \widetilde{C}^{8}$ is a $\mathbb{P}^{1}$-bundle over $\widetilde{C}^{8}$.

(3) Each fiber of $f$ is a rational elliptic surface with global sections.

(4) Let $V$ be the strict transform of $\widetilde{C} \times \widetilde{C}^{8}\left(\subset \mathbb{P}^{2} \times \widetilde{C}^{8}\right)$ in $X$. Then $s:=\tau(V)$ is a section of $q: W \rightarrow \tilde{C}^{8}$ and we have $\left.\tau^{-1}(\jmath) \simeq\right\lrcorner \times \tilde{C}$.

By our construction, $\tau$ has a global section, which is a $(-1)$-curve when restricted to each fiber of $f$ and the discriminant locus of $\tau$ do not intersect $\delta$. 
Take a sufficiently fine, finite open covering $\left\{U_{\alpha}\right\}$ of $\tilde{C}^{8}$ and a point $p_{\alpha}$ on $U_{\alpha}$ such that $q^{-1}\left(U_{\alpha}\right) \simeq U_{\alpha} \times \mathbb{P}^{1}$. Then we can perform generalized logarithmic transformations of multiplicity $m$ along $\left.{ }^{\prime}\right|_{U_{\alpha}}$ (cf. [2] [8]) and we have the following commutative diagram:

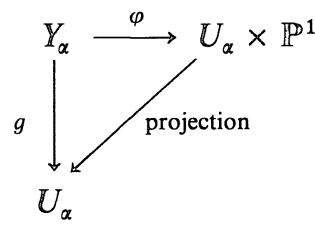

where

(1) $\varphi: Y_{\alpha} \rightarrow U_{\alpha} \times \mathbb{P}^{1}$ is an elliptic fiber space which has multiple fibers of multiplicity $m$ along $\left.{ }^{\prime}\right|_{U_{\alpha}}$.

(2) $g$ is smooth and each fiber of $g$ is a rational elliptic surface of with a multiple fiber of type ${ }_{m} I_{0}$.

(3) $\varphi^{-1}(\jmath) \simeq \jmath \times C$.

(4) For all $p \in \delta$, we have $\left.[\varsigma \times C]\right|_{\varphi^{-1}(p)} \simeq \eta$ in $\operatorname{Pic}^{o}(C)$.

By proposition (1.1.), $S_{\alpha}:=g^{-1}\left(p_{\alpha}\right)$ is a nine-points-blowing-up of $\mathbb{P}^{2}$.

Let $S_{\alpha} \stackrel{\sigma_{1}}{\rightarrow} S_{\alpha}^{1} \stackrel{\sigma_{2}}{\rightarrow} \cdots \stackrel{\sigma_{8}}{\rightarrow} S_{\alpha}^{8} \stackrel{\sigma_{9}}{\rightarrow} S_{\alpha}^{9} \simeq \mathbb{P}^{2}$ be the succession of blowing-downs and let $e^{(i)}$ be the exceptional curve of $\sigma_{i}: S_{\alpha}^{(i-1)} \rightarrow S_{\alpha}^{i}$. Then from the stability of the exceptional curves of the first kind, we may assume that there exists an irreducible divisor $D$ of $Y_{\alpha}$ which satisfies the following conditions.

(1) $g^{-1}\left(p_{\alpha}\right) \cap D \simeq e^{(1)}$.

(2) For all $t \in U_{\alpha}, g^{-1}(t) \cap D$ is an exceptional curve of the first kind on $g^{-1}(t)$.

By Remark (1.2.), $D$ is a multi-section of $\varphi: Y_{\alpha} \rightarrow \mathbb{P}^{1} \times U_{\alpha}$, which ramifies along $\left.\right|_{U_{\alpha}}$ with the ramification index $m$. Hence $\varphi$ and $g$ are locally projective. Let $H$ be a relatively ample divisor of $g: Y_{\alpha} \rightarrow U_{\alpha}$ and let $m$ be the self-intersection number $(D \cap$ $\left.g^{-1}(t), H \cap g^{-1}(t)\right)$ on $g^{-1}(t)$. For a sufficiently large positive integer $k$, we have the following commutative diagram.

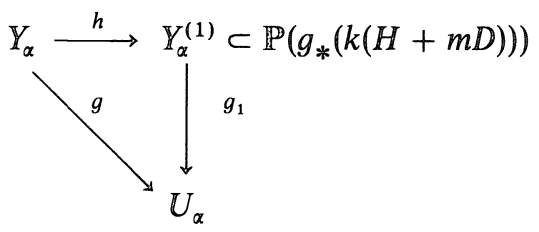

Then for each $t \in U_{\alpha}, h$ and $g_{1}$ are morphisms and $h_{t}: g^{-1}(t) \rightarrow g_{1}^{-1}(t)$ is a contraction morphism, that is $h_{t}\left(g^{-1}(t) \cap D\right)$ is a point. Continuing analogously, we finally obtain a commutative diagram:

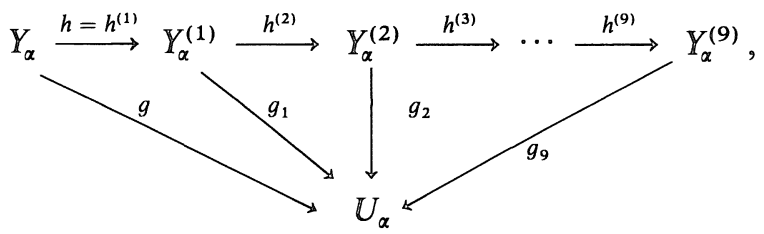

where $g_{9}^{-1}\left(p_{\alpha}\right) \simeq \mathbb{P}^{2}$ 
Since the deformation of $\mathbb{P}^{2}$ is isomorphic to $\mathbb{P}^{2}$, for all $t \in U_{\alpha}$, we have $g_{9}^{-1}(t) \simeq \mathbb{P}^{2}$ and $g^{-1}(t) \stackrel{h_{t}=h_{t}^{(1)}}{\rightarrow} g_{1}^{-1}(t) \stackrel{h_{1}^{(2)}}{\rightarrow} g_{2}^{-1}(t) \rightarrow g_{2}^{-1}(t) \rightarrow \cdots \stackrel{h_{9}^{(9)}}{\rightarrow} g_{9}^{-1}(t) \simeq \mathbb{P}^{2}$ is a succession of blowingdowns. By taking $U_{\alpha}$ smaller if necessary, we may assume that $Y_{\alpha}^{(9)} \simeq U_{\alpha} \times \mathbb{P}^{2}$ and $g_{9}^{-1}(\jmath) \simeq \triangleleft \times C$.

Put $\Psi:=h^{(9)} \circ h^{(8)} \circ \cdots \circ h^{(1)}$ and let $\Delta_{i}$ be nine irreducible exceptional divisors of $\Psi: Y_{\alpha} \rightarrow Y_{\alpha}^{(9)}$. By $\Psi$, each fiber of $\left.g\right|_{\Delta_{\alpha}}$ are mapped to a point on $\sigma \times C$. Hence there exists a morphism $\tilde{\phi}_{i}^{(\alpha)}: U_{\alpha} \rightarrow \Im \times C$ such that we have the following commutative diagram:

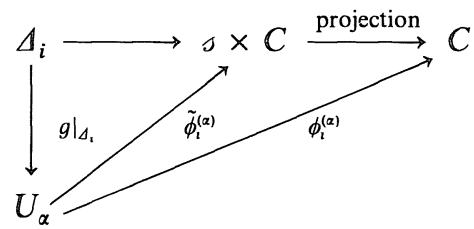

Note that if the two non-singular cubics in $\mathbb{P}^{2}$ are isomorphic, they are projectively equivalent.

Hence from the necessary condition in Theorem (2.1.) and the continuity of $\phi_{i}^{(\alpha)}$, there exists a morphism

$$
\phi^{(\alpha)}:=\left(\phi_{1}^{(\alpha)}, \ldots, \phi_{9}^{(\alpha)}\right): U_{\alpha} \rightarrow M:=\left\{\left(z_{1}, \ldots, z_{9}\right) \in C^{9} \mid \sum_{i=1}^{9} z_{i}=\eta\right\} .
$$

Now, we need the following lemma.

Lemma (2.4.) Let $(C, Q)$ and $\varepsilon: C \simeq T$ be as in the assumption of Theorem (2.1.). Assume that the surface $S_{1}$ (resp. $S_{2}$ ) obtained by blowing up $\mathbb{P}^{2}$ at nine points $p_{1}, \ldots, p_{9}$ (resp. $Q_{1}, \ldots, Q_{9}$ ) on $C$ has the structure of a rational elliptic surface with multiple fibers $m E_{i}(i=1,2)$ and they are analytically isomorphic. Then $P_{i}^{\prime}$ s and $Q_{i}$ 's $(1 \leq i \leq 9)$ are projectively equivalent.

Moreover, if we identify $E_{1}$ with a complex torus $T$ and $\left.\varphi\right|_{E_{1}}$ is expressed in the form: $z \rightarrow \alpha z+\beta, \alpha, \beta \in \mathbb{C}$, then $\beta$ is a three-torsion point of $T$ and we have $\alpha \eta=\eta$, where $\eta:=\sum_{i=1}^{9} \varepsilon\left(p_{i}\right)$ is an m-torsion point of $T$.

Proof of lemma (2.4.) Assume that there exists an isomorphism

$$
\varphi: S_{1} \simeq S_{2} .
$$

Since $E_{i} \in\left|-K_{S_{1}}\right|$ is the fixed component of the anti-canonical system $\left|-K_{S_{l}}\right|$, we have $\varphi\left(E_{1}\right)=E_{2}$. Hence by Demazure-Pinkham [1] and Looijenga [4], $P_{i}$ 's and $Q_{i}$ 's are projectively equivalent.

Since a line in $\mathbb{P}^{2}$ is mapped to a line in $\mathbb{P}^{2}$, we have $3 \beta=0$. Moreover, since we have an isomorphism: $\varphi^{*}: N_{E_{2} / S_{2}} \simeq N_{E_{1} / S_{1}}, \alpha$ must satisfy the following relation:

$$
\sum_{i=1}^{9}\left(\alpha \varepsilon\left(p_{i}\right)+\beta\right)=\sum_{i=1}^{9} \varepsilon\left(p_{i}\right)=: \eta
$$

Because $\beta$ is a three-torsion point of $T$, we have $\alpha \eta=\alpha$.

q.e.d. 
Now, let us continue the proof of Theorem (2.1.). Let $G$ be a finite group of automorphisms of $T$ generated by an element in the following form: $z \rightarrow \alpha z+\beta$, where $\beta$ is a three-torsion point of $T$ and $\alpha \eta=\eta$.

From the construction of $G$ and $M, G$ and the permutation groups $\mathscr{S}_{9}$ act on $M$ properly discontinuously and let $\bar{M}$ be its quotient space. Clearly $\bar{M}$ is a complete irreducible algebraic variety of dimension eight. By $\overline{\left(z_{1}, \ldots, z_{9}\right)}$, we denote the point of $\bar{M}$ corresponding to a point $\left(z_{1}, \ldots, z_{9}\right) \in M$. Let us consider a morphism

$$
\begin{aligned}
\overline{\phi^{(\alpha)}:} U_{\alpha} & \rightarrow \bar{M} \\
& \Psi \\
z & \rightarrow \overline{\left(\phi_{1}^{(\alpha)}(z), \ldots, \phi_{9}^{(\alpha)}(z)\right) .}
\end{aligned}
$$

By Lemma (2.4.), $\left\{\overline{\phi^{(\alpha)}}\right\}_{\alpha}$ defines a morphism $\bar{\Phi}: \widetilde{C}^{8} \rightarrow \bar{M}$ and $\bar{\Phi}$ is independent of the choice of $P_{\alpha} \in U_{\alpha}$. Since the dimension of the moduli of $Y_{\alpha} \rightarrow U_{\alpha}$ is eight, each fiber of $\bar{\Phi}$ must be discrete and $\bar{\Phi}$ is finite. Hence $\bar{\Phi}$ is surjective, and Theorem (2.1.) follows from Lemma (2.4.).

q.e.d.

Corollary (2.5.) Let $\Gamma$ be the subset of $\left(\mathbb{P}^{2}\right)^{9}$ consisting of $\left(p_{1}, \ldots, p_{9}\right)$ such that the following condition is satisfied.

Condition $(B)$. The rational surface $S$, obtained by blowing up $\mathbb{P}^{2}$ at nine points $p_{1}, \ldots, p_{9}$, has the structure of an elliptic surface.

Then $\Gamma$ is dense in $\left(\mathbb{P}^{2}\right)^{9}$ in the complex topology.

Proof. This follows immediately from Theorem (2.1.).

Remark (2.6.) In view of Proposition (1.1.), we have at the same time shown the existence of singular curves of degree $3 \mathrm{~m}$ which has an ordinary singularity of multiplicity $m$ at each $p_{i}(1 \leq i \leq 9)$ and is non-singular except them, under the assumption that the nine points $p_{1}, \ldots, p_{9}$ are mutually distinct.

\section{§3. Construction of $\mathbb{F}$ Fmily of Rational Elliptic Surfaces with Multiple Fibers}

In this section, we shall construct a family of rational elliptic surfaces with multiple fibers after Naruki's method. (cf. [6] §2,3.)

Example (3.1.) Let $T$ be a non-singular elliptic curve with the period $(1, \tau), \tau \in \mathbb{C}$, $\operatorname{Im}(\tau)>0$. Fix a polarization

$$
\begin{aligned}
\varepsilon: T & \rightarrow C_{\infty} \subset \mathbb{P}^{2} \\
\Psi & \Psi \\
t & \rightarrow\left(\mathscr{P}(t): \mathscr{P}^{\prime}(t): 1\right) \quad \text { if } t \neq 0 \\
0 & \rightarrow(0: 1: 0)=: Q \quad \text { if } t=0,
\end{aligned}
$$

where $C_{\infty}$ is a non-singular cubic curve in $\mathbb{P}^{2}$ with the fixed inflexion point $Q$. Let $C$ be the tangent line of $C_{\infty}$ at $Q$ and let $l_{t}(t \in T)$ be the line in $\mathbb{P}^{2}$ passing through two points $Q$ and $\varepsilon(t)$.

The pencil $L_{t}$ generated by $C_{\infty}$ and $2 C+l_{t}$ has an $A_{6}$-singularity over $Q$ and its 
minimal resolution is a rational elliptic surface with global sections, which has a singular fiber of type III* (resp. II*) over $0 \in \mathbf{P}^{1}$, if $t \neq 0$ (resp. if $t=0$ ).

Let $\left\{\theta_{1}, \theta_{2}\right\}$ be a basis of $H^{0}\left(C_{\infty}, \mathcal{O}(2 Q)\right)$, which can be expressed by using a Riemann-theta function of degree two. Then we have $\mathscr{P}(t)=\theta_{1}(t) / \theta_{2}(t)$ and $l_{t}$ can be defined by $l_{t}: \theta_{2}(t) \cdot x-\theta_{1}(t) \cdot z=0$. Next, put $S:=\mathbb{P}\left(\mathcal{O}_{T}(2 Q) \oplus \mathcal{O}_{T}\right)$ and take a sufficiently fine open covering $\left\{U_{i}\right\}$ of $T$ with local coordinate $\left(t_{i}, \zeta_{i}\right)$ such that $\zeta_{i}$ is a fiber coordinate of $S$. Note that the complete linear system $|2 Q|$ is base point free.

Then

$$
W:=\left\{\zeta_{i} f_{\infty}(x, y, z)+z^{2}\left(\theta_{2}^{(i)}(t) \cdot x-\theta_{1}^{(i)}(t) \cdot Z\right)=0\right\} \subset S \times \mathbb{P}^{2}
$$

is the one-parameter family of $L_{t}$ parameterized by $t \in T$. By Naruki [6], $\S 3$, we can construct the simultaneous resolution of $W \rightarrow T$ without base changes and get the following commutative diagram

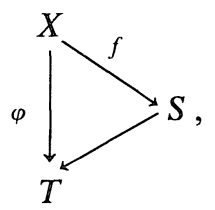

where

(1) $f: X \rightarrow S$ is an elliptic threefold over $S$ and has a regular fiber $C_{\infty}$ (resp. a singular fiber of type III*) along the infinite section (resp. the zero section) of $S$.

(2) $\varphi$ is smooth and each fiber of $\varphi$ is a rational elliptic surface with global sections as above.

By taking an $m$-sheeted ètale covering $\tilde{T}$ of $T$ and pulling back the family to $\widetilde{T}$, we get $\tilde{X} \rightarrow \widetilde{S} \rightarrow \widetilde{T}$.

Note that the self-intersection number of the infinite section $\tilde{J}_{\infty}$ of $\tilde{S}$ is divisible by $m$. Hence by Fujimoto [2], theorem (2.1.), we can perform generalized logarithmic transformations of degree $m$ along $\tilde{J}_{\infty}$ and get the following commutative diagram.

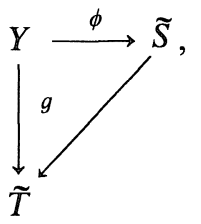

where

(1) $\phi: Y \rightarrow \widetilde{S}$ is an elliptic fiber space which has multiple fibers of multiplicity $m$ along $\tilde{J}_{\infty}$,

(2) $g$ is smooth and each fiber of $g$ is a rational elliptic surface with multiple fibers.

Remark (3.2.) The total space $Y$ is not in the class $\mathscr{C}$ (see Fujimoto [2], remark (2.5)). However $\phi$ is locally projective, as is clear from the proof of theorem (2.1.).

Example (3.3.) Let $\varepsilon: T \simeq\left(C_{\infty}, Q\right)$ be as in example (3.1.) and $\left\{\theta_{1}, \theta_{2}, \theta_{3}\right\}$ be a basis of $H^{0}\left(C_{\infty}, \mathcal{O}(3 Q)\right)$ such that

$$
\mathscr{P}(t)=\frac{\theta_{1}(t)}{\theta_{2}(t)}, \mathscr{P}^{\prime}(t)=\frac{\theta_{2}(t)}{\theta_{3}(t)}
$$


Put $M=\left\{\alpha=\left(\alpha_{1}, \ldots, \alpha_{9}\right) \in(T)^{9} \mid \sum_{i=1}^{9} \alpha_{i}=0\right\}$. By Theorem (2.1.) (1), for all $\alpha \in M$, there exists a pencil of cubic curves in $\mathbb{P}^{2}$ passing through nine points $\left(\varepsilon\left(\alpha_{i}\right)\right)_{1 \leq i \leq 9}$ on $C_{\infty}$.

For $\alpha=\left(\alpha_{1}, \ldots, \alpha_{9}\right) \in M$, we set $g_{\alpha}(t):=\prod_{i=1}^{9} \theta\left(t-\alpha_{i}\right)$, where $\theta(t) \in H^{0}(T, \mathcal{O}(Q))$ is the Riemann theta function of degree one on $T$ with a zero of order one at $t=0$ and $\theta_{3}=\theta^{3}$. Then from the choice of $\alpha$, we have $g_{\alpha}(t) \in H^{0}(T, \mathcal{O}(9 Q))$. We choose a basis $\left\{\sigma_{i}(t)\right\}_{1 \leq i \leq 9}$ of $H^{0}(T, \mathcal{O}(9 Q))$ as follows.

$$
\left(\sigma_{1}, \sigma_{2}, \sigma_{3}, \sigma_{4}, \sigma_{5}, \sigma_{6}, \sigma_{7}, \sigma_{8}, \sigma_{9}\right):=\left(\theta_{3}{ }^{3}, \theta_{1} \theta_{3}{ }^{2}, \theta_{2} \theta_{3}{ }^{2}, \theta_{1}{ }^{2} \theta_{3}, \theta_{1} \theta_{2} \theta_{3}, \theta_{1}{ }^{3}, \theta_{1}{ }^{2} \theta_{2}, \theta_{1} \theta_{2}{ }^{2}, \theta_{2}{ }^{3}\right)
$$

Then we have

$$
\left(\sigma_{1}(0), \ldots, \sigma_{9}(0)\right)=(0,0,0,0,0,0,0,0,1)
$$

and

$$
\varepsilon^{*}\left(\sigma_{1}, \sigma_{2}, \sigma_{3}, \sigma_{4}, \sigma_{5}, \sigma_{6}, \sigma_{7}, \sigma_{8}, \sigma_{9}\right)=\left(z^{3}, x z^{2}, y z^{2}, x^{2} z, x y z, x^{3}, x^{2} y, x y^{2}, y^{3}\right)
$$

Then $g_{\alpha}(t)$ can be written as a linear combination of $\sigma_{i}$ 's:

$$
g_{\alpha}(t)=\sum_{i=1}^{9} m_{i} \sigma_{i}(t), \quad m_{i} \in \mathbb{C} .
$$

Now we show that $m_{i}$ 's can be expressed as a meromorphic function of $\alpha \in M$. By noting $\theta(0)=0$ and substituting $t=\alpha_{1}, \ldots, \alpha_{8}, 0$ to $(*)$, we have the following equation.

$$
\left(\begin{array}{c}
\sigma_{1}\left(\alpha_{1}\right), \sigma_{2}\left(\alpha_{1}\right), \ldots, \sigma_{9}\left(\alpha_{1}\right) \\
\ldots \ldots \ldots \ldots \ldots \ldots \ldots \ldots \ldots \\
\sigma_{1}\left(\alpha_{i}\right), \sigma_{2}\left(\alpha_{i}\right), \ldots, \sigma_{9}\left(\alpha_{i}\right) \\
\ldots \ldots \ldots \ldots \ldots \ldots \ldots \ldots . \\
\sigma_{1}\left(\alpha_{8}\right), \sigma_{2}\left(\alpha_{8}\right), \ldots, \sigma_{9}\left(\alpha_{8}\right) \\
0, \quad 0, \ldots, \quad 1
\end{array}\right)\left(\begin{array}{c}
m_{1} \\
m_{i} \\
m_{8} \\
m_{9}
\end{array}\right)=\left(\begin{array}{c}
0 \\
\vdots \\
\vdots \\
0 \\
\beta
\end{array}\right), \quad \text { where } \beta:=\prod_{i=1}^{9} \theta\left(\alpha_{i}\right) .
$$

Let $D$ be the $9 \times 9$ matrix defined on the left hand side and let $P_{j}$ be the determinant of a $8 \times 8$ matrix obtained from $D$ by deleting the 9-th row and the $j$-th column. Then from an easy calculation, we have

$$
\operatorname{det}(D)=P_{9}, \quad m_{9}=\beta \quad \text { and } \quad m_{j}=(-1)^{j} \beta \frac{P_{j}}{P_{9}} .
$$

Hence we have $P_{9} \prod_{i=1}^{9} \theta\left(t-\alpha_{i}\right)=\beta \sum_{i=1}^{9}(-1)^{i} P_{i}\left(\alpha_{1}, \ldots, \alpha_{9}\right) \sigma_{i}(t) . \quad$ Each $P_{i}=P_{i}\left(\alpha_{1}, \ldots\right.$, $\alpha_{9}$ ) vanishes at $\alpha_{k}=\alpha_{l}(1 \leq k \leq l \leq 9)$, so by subtracting the common zeros of $P_{i}^{\prime}$ 's, we may assume that any two of $P_{i}^{\prime}$ 's are relatively prime and $P_{i} \in H^{0}(M, \mathcal{O}(L))$ for some line

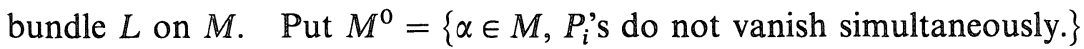

Let $C_{\alpha}\left(\alpha \in M^{0}\right)$ be the cubic curve in $\mathbb{P}^{2}$ defined as follows.

$$
\widehat{C}_{\alpha}: f_{\alpha}:=P_{1} z^{3}-P_{2} x z^{2}+P_{3} y z^{2}-P_{4} x^{2} z+P_{5} x y z-P_{6} x^{3}+P_{7} x^{2} y-P_{8} x y^{2}+P_{9} y^{3}=0
$$

Then $C_{\alpha}$ intersects $C_{\infty}$ with the nine points $\varepsilon\left(\alpha_{i}\right)(i=1, \ldots, 9)$. 
Next, put $V:=\mathbb{P}\left(\mathcal{O}_{M} \oplus \mathcal{O}_{M}(L)\right)$ and take a fine open covering $\left\{U_{i}\right\}$ of $M$ with the local coordinate $\left(t_{i}, \zeta_{i}\right)$ such that $\zeta_{i}$ is the fiber coordinate of $V$. Then $W:=$ $\left\{\zeta_{i} f_{\infty}(x, y, z)+f_{\alpha}(x, y, z)=0\right\}$ is the one-parameter family of the pencil $\left[C_{\infty}, C_{\alpha}\right]$ parameterized by $\alpha \in M^{0}$. As in Example (3.1.), we can construct the simultaneous resolution of $W$ and by the generalized logarithmic transformations along the infinite section of $V$, we get a family of rational elliptic surfaces with multiple fibers over an 8-dimensional (non-compact) complex manifold.

\section{References}

[1] Demazure, M., Pinkham, H. and others, Seminaire sur les singularités des surfaces, Springer L. N., 777 (1980), Berlin-Heidelberg-New York.

[2] Fujimoto, Y., Logarithmic transformations on elliptic fiber spaces, J. of Math. Kyoto Univ., 28 (1988), 91-110.

[3] Kodaira, K., On compact analytic surfaces I-III, Ann. of Math., 71 (1960), 111-152, 77 (1963), 523-626, 78 (1963), 1-40.

[4] Looijenga, E., Rational surfaces with anti-canonical cycle, Ann. of Math. (2), 114 (1981), 267-322.

[5] Naruki, I., Configurations related to maximal rational elliptic surfaces, Complex Analytic Singularities, Advanced Studies in Pure Math., 8 (1986), 314-340, Kinokuniya.

[6] - K3 surfaces related to root systems in $E_{8}$, (to appear in Prospects of Algebraic Analysis.)

[7] Persson, U., Double sextics and singular K3 surface, Algebraic Geometry, Proceeding, Springer L. N., 1124 (1985), 262-328.

[8] Ueno, K., Degeneration of elliptic surfaces and certain non-Kähler manifolds, Progress in Math., 39 (1983), 545-566. 
